\title{
Nanobody-Functionalized Cellulose for Capturing and Containing SARS-CoV-2
}

\author{
Xin Sun ${ }^{1}$, Shaobo Yang ${ }^{1}$, Amal AI-Dossary ${ }^{2}$, Shana Broitman ${ }^{1}$, Yun Ni ${ }^{1}$, Mengdi Yang ${ }^{1}$, \\ and Jiahe $\mathrm{Li}^{1}$ \\ ${ }^{1}$ Northeastern University \\ ${ }^{2}$ Imam Abdulrahman Bin Faisal University
}

August 4, 2021

\begin{abstract}
The highly transmissible severe acute respiratory syndrome coronavirus 2 (SARS-CoV-2) has infected more than 196 million people, claiming 4.2 million lives to date. Although mandatory quarantines, lockdowns, and vaccinations help curb viral transmission, safe and effective preventative measures remain urgently needed. Here, we present a generic strategy for containing SARS-CoV-2 by cellulose materials. Specifically, we developed a bifunctional fusion protein consisting of a cellulose-binding domain and a nanobody $(\mathrm{Nb})$ targeting the receptor-binding domain of SARS-CoV-2. The immobilization of the fusion proteins on cellulose substrates enhanced the capture efficiency of Nbs against SARS-CoV-2 pseudoviruses of both the wildtype and the D614G variant, the latter of which has been shown to confer higher infectivity. Furthermore, the fusion protein was integrated into a customizable chromatography with highly porous cellulose for neutralizing virus from contaminated fluids in a continuous and cost-effective fashion. Taken together, our work leverages low-cost cellulose materials and recently developed Nbs to provide a complementary approach to addressing the pandemic.
\end{abstract}

\section{Introduction}

Since the first documented coronavirus disease 2019 (COVID-19) case at the end of 2019 (Liu et al., 2020), the highly contagious severe acute respiratory syndrome coronavirus 2 (SARS-CoV-2) has resulted in at least 196 million positive cases and 4.2 million deaths ( $2.17 \%$ fatality rate) in 219 countries and territories (as of July 28, 2021, source:https://covid19.who.int/). To contain the spread of SARS-CoV-2, non-pharmaceutical interventions were originally deployed, including the employment of masks, handwashing, and public measures such as city lockdowns, travel restrictions, and social distancing. However, the long-term adherence to these preventative measures has led to severe societal and economic crises (Lai et al., 2020). Importantly, the approval and administration of several SARS-CoV-2 vaccines worldwide has helped to mitigate the pandemic waves with an ever-increasing immunization population (Haghpanah et al., 2021). Nevertheless, COVID-19 poses a continued threat because of constantly emerging SARS-CoV-2 variants and relatively long duration for herd immunity (Krause et al., 2021). Therefore, there is a great demand for effective, low-cost, and offthe-shelf agents to fast diagnose and decontaminate SARS-CoV-2 from body fluids and frequently contacted environmental surfaces (Ong et al., 2020).

SARS-CoV-2 belongs to the $\beta$-sopovaıvs genus of the Coronaviridae family, and shares the same subfamily Orthocoronaviridae with SARS-CoV, all of which lead to severe respiratory tract illness in humans (Zhou et al., 2020). SARS-CoV-2 is a single-stranded RNA composed of $30 \mathrm{~kb}$ nucleotides, which encode four major structural proteins: the spike $(\mathrm{S})$, the membrane $(\mathrm{M})$, the envelope(E), and the nucleocapsid $(\mathrm{N})$ (Luan et al., 2020). Viral infections rely upon cellular entry to utilize the host's machinery for replicating viral copies that are then released by the host. The S protein facilitates the attachment of the virus to the host's cellular 
receptors and promotes the fusion between host and viral membranes (Schoeman \& Fielding, 2019). In particular, the S protein contains the receptor-binding domain (RBD), which binds to the extracellular domain of the host receptor angiotensin-converting enzyme 2 (ACE2) for viral entry (Bangaru et al., 2020; Lan et al., 2020; Sternberg \& Naujokat, 2020; Wrapp et al., 2020). Recent work demonstrates that SARS-CoV-2 targets the same functional host receptor, ACE2, as SARS-CoV (W. Li et al., 2003; Luan et al., 2020; Zhou et al., 2020). However, SARS-CoV-2's RBD is 10 - to 20-fold higher than that of SARS-CoV in ACE2 binding (Kirchdoerfer et al., 2018). Owing to the key roles of SARS-CoV-2's S protein or the subdomain RBD in the entry to host cells, the S protein or RBD has been extensively explored as a key target for the development of antiviral antibodies, among which nanobodies (Nbs) represent a unique class towards these efforts. Nbs are single-domain nanosized antibodies, which are derived from variable fragments ofCamelidae (including camels and llamas) heavy chain-only antibodies (hcAbs) (Hassanzadeh-Ghassabeh et al., 2013; Steeland et al., 2016). Nbs offer a variety of advantages over other antibodies for diagnostic development: (i) nanometer size, (ii) high affinity and specificity, (iii) deep penetration in tissues, (iv) low immunogenicity, (v) easy scalability for mass production, and (vi) thermostability (Steeland et al., 2016; Zhao et al., 2018). Because of these benefits, to date, several high-affinity neutralizing Nbs directed against SARS-CoV-2's S protein and RBD domain have been identified, among which Ty1 has shown nanomolar binding affinity and effective neutralization through immunization in alpaca followed by the phage display (Hanke et al., 2020; Huo et al., 2020; Koenig et al., 2021; Schoof et al., 2020; Xu et al., 2021). It was found that Ty1 specifically targets the RBD of SARS-CoV-2 with high affinity and directly blocks ACE2 engagement. Therefore, Ty1 can serve as a potential biologic for various diagnostic and therapeutic applications against COVID-19.

In this study, we repurposed the Nb Ty1 to detect and neutralize SARS-CoV-2 on surfaces and in biologically relevant fluids in a low-cost platform based on cellulose materials. Specifically, we designed a bifunctional fusion protein that comprises of a cellulose-binding domain (CBD) and Ty1 for cellulose immobilization and SARS-CoV-2 capturing, respectively. (S. Kim et al., 2021) The CBD originating from the cip $A$ gene in the bacterium $C$. thermocellum has been shown to be resistant to heat denaturation $\left(\mathrm{Tm} \geq 70{ }^{\circ} \mathrm{C}\right)$ due to the thermophilic nature of $C$. thermocellum (Voutilainen et al., 2014). Additionally, Nbs are generally more thermostable and easier to manufacture (e.g., E. coli fermentation) than conventional human immunoglobin (IgG) based antibodies, the latter of which require mammalian cell hosts for production (Harmsen \& De Haard, 2007). As a result, our fusion technology is highly cost-effective, thermostable, and scalable to overcome various challenges posed by the pandemic, including but not limited to, disrupted supply chains, restricted deployment to remote areas, and mass production. As a proof-of-concept, we performed an immunoassay on cellulose-based filter paper for detecting SARS-CoV-2's RBD using our bifunctional proteins. Furthermore, we developed a customized cellulose-based affinity chromatography to remove SARS-CoV-2 viral particles from biologically relevant fluids using pseudoviruses of both wildtype and the D614G variant, which may pave the way for decontaminating blood products and other liquids (Chang et al., 2020; Ragan et al., 2020). Given the modularity of our bifunctional protein platform and the ease of rapidly identifying target-specific Nbs through immunization and directed evolution, our work can potentially provide a framework to address other emerging infectious diseases with similar approaches.

\section{Materials and Methods}

\section{Reagents and chemicals}

Tween-20, Triton X-100 (TX-100) and Triton X-114 (TX-114) were obtained from Sigma-Aldrich (St Louis, MO). Strep-tag and Strep-Tactin XT were purchased from IBA Lifesciences (Gottingen, Germany). Detergent compatible (DC) protein assay kit was bought from Bio-Rad Laboratories (Hercules, CA). Anti-FLAG epitope (DYKDDDDK, catalog\# 637301) and horseradish peroxidase (HRP) Donkey anti-human IgG antibody (catalog \#410902) were purchased from Biolegend (San Diego, CA). The secondary antibody anti-rat IgG HRP (catalog\# 7077) was bought from Cell Signaling Technology (CST, Danvers, MA). All other reagents and chemicals, including nickel nitrilotriacetic acid (Ni-NTA) agarose and pierce rapid gold protein 
assay kit were purchased from Fisher Scientific International Inc., (Hampton, NH), and were of highest purity or analytical grade commercially available.

Cell lines

HEK293T cells expressing human angiotensin I-converting enzyme 2 (HEK293T-hACE2) were kindly provided by Dr. Jesse Bloom (Fred Hutchinson Cancer Research Center, Seattle, USA) (Crawford et al., 2020). Lenti-X 293T cell line was purchased from Takara Bio USA Inc. (San Jose, CA). These cell lines were maintained in complete Dulbecco's modified Eagle's medium (DMEM) (Corning, NY) supplemented with 10\% fetal bovine serum (FBS; Corning, NY) and $100 \mathrm{U} / \mathrm{ml}$ penicillin-streptomycin (Corning, NY) at $37 @ \mathrm{C}$ in humidified incubator with $5 \%$ carbon dioxide $\left(\mathrm{CO}_{2}\right)$. Cells at passages 2-10 were used for the experiments.

\section{Plasmids construction, protein expression and purification}

Ty1 variants, including Ty1-CBD and control protein Ty1 without CBD module, were cloned into pSH200 vector (a generous gift from Prof. Xiling Shen at Duke University) containing 6 x Histidine tag (His-tag), between BamHI and XbaI sites. Both plasmids were validated by sequencing before expression. Ty1-CBD and Ty1 were expressed and produced as previously described (Yang et al., 2020; Sun et al., 2021). Produced protein was sequentially purified by affinity chromatography using Ni-NTA agarose beads and fast protein liquid chromatography (FPLC, NGC Quest 10 Chromatography system, Biorad, Hercules, CA). Protein fractions detected at $\lambda=280$ were collected. Collected protein fraction were quantified by DC protein assay and purities were verified by sodium dodecyl sulfate-polyacrylamide gel electrophoresis (SDS-PAGE). Validated protein was aliquoted and kept at $-80 @ \mathrm{C}$ with 50\% glycerol at all times until future use.

\section{Cellulose paper-based immunoblotting}

To validate the binding ability of Ty1-CBD, purified Ty1-CBD was spotted on cellulose paper and dried at room temperature. Cellulose paper with dried Ty1-CBD were incubated with $5 \mathrm{ml}$ of $5 \%$ nonfat milk in Tris-buffered saline (TBS) for 30 min to block non-specific binding sites. After blocking, the cellulose paper was then incubated with anti-FLAG epitope (DYKDDDDK), which was diluted at 1:2000 in $3 \mathrm{ml}$ TBS plus $5 \%$ nonfat milk, overnight at $4^{\circ} \mathrm{C}$. The paper was washed three times with $5 \mathrm{ml} 1 \mathrm{xTBS}$ containing $0.05 \%$ Tween-20 (TBST), with 15 min per wash cycle. The paper was incubated with HRP anti-rat IgG (1: 2000) for $1 \mathrm{hr}$ at room temperature. After washing with 1xTBS with $5 \mathrm{ml} 0.05 \%$ Tween-20, premixed Pierce 3,3'diaminobenzidine (DAB) substrate was directly added on the cellulose paper. The reaction was terminated with water after dark spots appeared.

\section{Pseudovirus production}

HDM-SARS2-Spike-delta21 and HDM-SARS2-Spike-del21-614G encoding SARS-CoV-2 Spike with 21 amino acid C-terminal deletion for lentiviral pseudo-typing were purchased from Addgene (Watertown, MA) with serial numbers of Addgene \#155130 and Addgene\#158762, respectively. Both plasmids were isolated following manufacturer's instructions and quantified through Nanodrop. $24 \mathrm{hr}$ prior to transfection, Lenti-X 293T cells in logarithmic growth phase were trypsinized, and the cell density was adjusted to $1.0 \times 10^{6}$ cells $/ \mathrm{mL}$ with complete DMEM medium. The cells were reseeded into a $10 \mathrm{~cm}$ cell culture dishes to reach $70 \%$ confluency on the day of transfection. The plasmid mixture was prepared according to Table $\mathbf{1}$ and Table $\mathbf{2}$. After inverting the plasmid mixture for 5-8 times, and kept at room temperature for $30 \mathrm{~min}$, the plasmid mixture was gently added to the Lenti-X 293T cells (Crawford et al., 2020). After $48 \mathrm{hr}$ and $72 \mathrm{hr}$ of transfection, the pseudovirus was collected and centrifuged at 1000xg at $4 @ \mathrm{C}$ for $20 \mathrm{~min}$ to remove the debris. The 
cell culture medium was replaced with fresh complete DMEM medium once the pseudovirus was collected. Aliquots of the harvested virus were stored at $4 @ \mathrm{C}$ for immediate use or frozen at $-80 @ \mathrm{C}$ for future use.

\begin{tabular}{ll}
\hline Composition & Amount \\
\hline HDM-SARS-2-Spike-delta21 (or D614G) & $2.5 \mu \mathrm{g}$ \\
pFuw-Ubc-GFP & $10 \mu \mathrm{g}$ \\
psPAX2 & $7.5 \mu \mathrm{g}$ \\
TransIT-X2 2000 & $30 \mu \mathrm{l}$ \\
Opti-MEM & $1000 \mu \mathrm{L}$ \\
\hline
\end{tabular}

Table 1 Compositions of the plasmid mixtures for generating SARS-CoV-2 pseudovirus (wildtype or D614G).

\begin{tabular}{ll}
\hline Composition & Amount \\
\hline pMD2.G & $2.5 \mu \mathrm{g}$ \\
pFuw-Ubc-GFP & $10 \mu \mathrm{g}$ \\
psPAX2 & $7.5 \mu \mathrm{g}$ \\
TransIT-X2 2000 & $30 \mu \mathrm{l}$ \\
Opti-MEM & $1000 \mu \mathrm{L}$ \\
\hline
\end{tabular}

Table 2: Compositions of the plasmid mixture for lentivirus generation as a positive control.

\section{Quantification of viral titers by flow cytometry}

HEK293T-hACE2 cells were seeded $24 \mathrm{hr}$ before the pseudovirus transduction assay in 96-well plates $\left(2 \times 10^{4}\right.$ cells/well in a volume of $100 \mu \mathrm{l}$ complete DMEM medium). On the co-culture day, the medium was removed and $200 \mu \mathrm{l}$ of prewarmed pseudovirus was added to the cells. Polybrene (Sigma Aldrich) was added into cultured HEK293T-hACE2 cells to a final concentration of $8 \mu \mathrm{g} / \mathrm{ml}$, to facilitate lentiviral infection through minimizing charge-repulsion between virus and cells. After transduction for $48 \mathrm{hr}$, cells were collected through trypsinization and transferred to a 96 well V-bottom plate with complete culture DMEM medium for neutralization. Cells were pelleted at $300 \mathrm{xg}$ for $3 \mathrm{~min}$ and washed twice with phosphate-buffered saline (PBS, $137 \mathrm{mM} \mathrm{NaCl}, 10 \mathrm{mM}$ phosphate, $2.7 \mathrm{mM} \mathrm{KCl}$; $\mathrm{pH}$ 7.4). After the final wash, the cells were resuspended in $200 \mu \mathrm{L}$ FACS buffer (5\% FBS, 2mM EDTA, $0.1 \%$ sodium azide in PBS) for flow cytometric analysis in Attune NxT Flow Cytometer (Thermofisher) and data were analyzed by FlowJo (Franklin Lakes, NJ). Using a well that has 1\%-20\% of GFP-positive cells, the titer was calculated according to the formula, titer $=\{(\mathrm{F} \times \mathrm{Cn}) / \mathrm{V}\} \times \mathrm{DF}, \mathrm{F}$ : The frequency of GFP-positive cells determined by flow cytometry; Cn: The total number of target cells infected; V: The volume of the inoculum. DF: The virus dilution factor. For all experiments triplicate samples were analyzed, data are representative of two or more experiments, and the standard error of the mean (SEM) is shown.

\section{Preparation of the regenerated amorphous cellulose $(R A C)$ co- lumn}

RAC was produced referring to RAC-based affinity protein purification as previously described (Yang et al., 2020). The RAC column was first equilibrated with 1xPBS and proteins of interest were added to the RAC column until the column was saturated by quantifying the amount of fusion protein in flow-through fractions and comparing it to the original supernatant via SDS-PAGE. The saturated RAC was washed 
with 10 column volumes of 1 xPBS followed by loading with $400 \mu$ l complete DMEM medium. $400 \mu \mathrm{L}$ of pseudovirus were flowed through the saturated RAC and collected for future use.

\section{Preparation of Ty1-CBD functionalized cellulose paper}

For capturing capability assay, cellulose filter paper discs were fitted into a 96 -well plate or $1.5 \mathrm{ml}$ microtube followed by blocking with $1 \%$ bovine serum albumin (BSA) in 1 x TBS for $1 \mathrm{hr}$. After aspirating the blocking buffer, $50-100 \mu \mathrm{l}$ purified proteins with concentrations of $100 \mu \mathrm{g} / \mathrm{ml}, 10 \mu \mathrm{g} / \mathrm{ml}$ and $1 \mu \mathrm{g} / \mathrm{ml}$ in $1 \times$ TBS or 400 $\mu \mathrm{l}$ protein lysate of interest were applied directly to the coated cellulose paper. After $1 \mathrm{hr}$ incubation at room temperature with slow shaking, purified proteins or lysates were removed from 96 well plates or 1.5 $\mathrm{ml}$ microtube, followed by 3 times wash with $1 \mathrm{x}$ TBS, $10 \mathrm{~min}$ in between. Cellulose paper was blocked with $5 \%$ nonfat milk in TBS at room temperature for $15-30$ min. $50-100 \mu \mathrm{L}$ media with spike expression were applied to the cellulose paper and incubated at room temperature for $1 \mathrm{hr}$. The treated cellulose paper was washed with TBST, 4 times, with 5-10 min each. The washed cellulose paper was incubated with HRP Donkey anti-human IgG antibody diluted in $1 \mathrm{x}$ TBS supplemented with $5 \%$ milk with antibody dilution of 1:2000. After $1 \mathrm{hr}$, the cellulose paper was washed with TBST for 4 times with $5-10$ each. Premixed DAB substrate was directly added on cellulose paper after removing the TBST. The reaction was terminated with distilled water till the color development.

For preparing the cellulose filter paper discs to evaluate the neutralization efficiency against the pseudovirus, cellulose filter paper discs were fitted into a 96-well or 48-well plate followed by blocking with $1 \%$ BSA in 1 $\mathrm{x}$ TBS for $1 \mathrm{hr}$. After aspirating the blocking buffer, $50-100 \mu \mathrm{l}$ of $10 \mu \mathrm{g} / \mathrm{ml}$ purified fusion proteins in $1 \mathrm{x}$ TBS were applied directly to the coated cellulose paper. After $1 \mathrm{hr}$ incubation at room temperature with slow shaking, diluted purified proteins were aspirated from 96-well or 48-well plates, followed by two times wash with $1 \mathrm{x}$ TBS, $10 \mathrm{~min}$ in between. 100-400 $\mu \mathrm{L}$ of pseudoviruses were added into each well with cellulose paper. After $1 \mathrm{hr}$ incubation, the cellulose paper treated pseudovirus was gently transferred to transfect HEK293T-hACE2 cells with $80 \%$ confluency, followed by adding polybrene to a final concentration of 8 $\mu \mathrm{g} / \mathrm{ml}$. $24 \mathrm{hr}$ post-transduction, HEK293T-hACE2 cells were split in a ratio of 1:3. $48 \mathrm{hr}$ post transfection, cells were collected at $300 \mathrm{xg}$ for $3 \mathrm{~min}$ followed by resuspending them in $200 \mu \mathrm{L}$ FACS buffer. Samples were loaded to Attune flow cytometry followed by analysis through FlowJo. After gating the HEK293T-hACE2 cells without pseudovirus (negative control) with less than $2 \%$ GFP positive, the transduction efficiency for experimental group is calculated by $\mathrm{GFP}^{+} /\left[\mathrm{GFP}^{+}+\mathrm{GFP}^{-}\right]$. For all experiments triplicate samples were analyzed, data are representative of two or more experiments, and the standard error of the mean (SEM) is shown.

\section{Statistical analysis}

Statistical significance was evaluated using one-way ANOVA followed by Tukey post hoc test using GraphPad PRISM (San Diego, CA, USA). Pvalues $<0.05$ were considered statistically significant. Statistical significance of our interest is indicated in all figures according to the following scale: ${ }^{*} \mathrm{P}<0.05,{ }^{*} \mathrm{P}<0.01$, ${ }^{* * *} \mathrm{P}<0.001$, and ${ }^{* * * *} \mathrm{P}<0.0001$. All graphs are expressed as the means $\pm \mathrm{SEM}$.

\section{Results}

\section{Genetic fusion of an RBD-specific nanobody with a cellulose-binding domain}

Our overall scheme for low-cost detection and neutralization of SARS-CoV-2 capitalizes on generating a bifunctional protein through the genetic fusion between the high-affinity Nb Ty1 targeting the RBD of SARS$\mathrm{CoV}-2$ and the thermostable cellulose-binding domain (CBD) (Figure 1 ). SARS-CoV-2 can be transmitted 
via airborne particles or through directly contacting contaminated surfaces (Morawska \& Cao, 2020). Therefore, considering that cellulose is prevalent in many materials, such as paper towels and the inner coating of face masks, we immobilized the fusion proteins to the surface of cellulose materials, such as filter paper, to enable viral detection or capturing of SARS-CoV-2 from the surface (Figure 1e ). Importantly, due to the specific interaction between the CBD domain and cellulose, we reason that the bifunctional Ty1-CBD can be immobilized in a defined orientation that favors the interaction between the $\mathrm{Nb}$ of interest and the antigen, as compared to random immobilization. On the other hand, viral transmission and contamination through blood products present a common issue during the pandemic (Ragan et al., 2020). Because blood products are susceptible to heat and chemical denaturation, it is desirable to devise a strategy that reduces and eliminates the viral load in blood products while maintaining the blood's bioactivities (Chang et al., 2020). To assess whether our strategy can address these challenges, we integrated the bifunctional fusion protein Ty1-CBD into a customized cellulose affinity purification column to allow for a target-specific depletion in a continuous manner (Figure 1f ).

\section{Production and purification of the bifunctional protein Ty1-CBD}

Compared to human IgG, Nbs can be produced in E. coli with high yield and purity (Figure 1a). Therefore, DNA encoding the fusion protein Ty1-CBD was first cloned in a standard expression vector for recombinant protein production in E. coli . Because the antigen-binding site of Nbs is closed to the $\mathrm{N}$ terminus, we placed the $\mathrm{CBD}$ at the $\mathrm{C}$ terminus of Ty1 to circumvent potential steric hindrance. Meanwhile, a short $6 \mathrm{x}$ histidine (His-tag) was attached to the $\mathrm{N}$ terminus for the metal affinity purification, while a FLAG epitope (DYKDDDDK) was inserted between Ty1 and the CBD, simultaneously serving as a hydrophilic flexible linkage and a tag for immunostaining (Figure 1b ). The yield of fusion proteins was estimated to be 50 mg per liter of bacterial culture in a shake-flask mode and was purified to high homogeneity as evidenced by denaturing SDS-PAGE and size exclusion chromatography (Figure 1c ,Figure 1d, Figure S1a, and Figure S1b ).

\section{The fusion protein is functionally active in cellulose binding and RBD detection}

Next, we sought to evaluate whether the fusion proteins were capable of cellulose binding and Nb-specific target recognition. To this end, we first spotted purified fusion proteins on the surface of cellulose paper (Figure 2a ). Upon air drying, the paper was stained with a rat antibody against the FLAG epitope in the fusion protein, followed by an anti-rat secondary antibody conjugated with horseradish peroxidase (HRP). A

dark precipitate was visualized after incubation with an HRP substrate, 3,3'-Diaminobenzidine (DAB). To quantify the binding efficiency of the fusion protein to the cellulose paper, we immobilized serially diluted fusion proteins within a defined area on the Whatman filter paper followed by immunostaining with an anti-FLAG antibody. Indeed, the extent of protein immobilization correlated with the staining in a certain concentration range (Figure $\mathbf{2 b}$ andFigure S1c ), from which we estimated that a surface area of $1 \mathrm{~mm}^{2}$ can be saturated by $500 \mathrm{ng}(\sim 0.02 \mathrm{nmol})$ of Ty1-CBD proteins in the Whatman filter paper.

Having validated the high binding capacity of purified Ty1-CBD to filter paper, we speculated that because the CBD itself can act as a natural affinity ligand to cellulose, we could directly immobilize $E$. coli cell lysate containing the CBD fusion proteins to filter paper, followed by extensive washing to remove nonspecific proteins. This circumvents the need to purify desired proteins beforehand, which is labor intensive and impractical when it comes to the large-scale manufacturing of functionalized cellulose materials (Figure 2c ). To this end, we first incubated $E$. coli cell lysate with filter discs and removed nonspecific proteins via washing. Then we subjected the functionalized filter discs to cell culture media containing secreted recombinant RBD as a proxy for actual SARS-CoV-2. As shown in Figure 2d, Ty1-CBD-coated filter paper was able to capture SARS-CoV-2's RBD as evidenced by the intense dark staining reflecting the detection of RBD. Of note, filter discs precoated with Ty1 alone exhibited light staining, likely due to nonspecific but weak absorption of Ty1 to cellulose. In comparison, in the control media without the RBD, neither Ty1- nor Ty1-CBD-coatd discs displayed the dark staining. Our findings here indicate that the CBD domain promoted the immobilization of Ty1-CBD on cellulose substrates while Ty1 remained able to specifically recognize the 
target.

\section{Immobilization of Ty1-CBD on filter paper increases the capture efficiency of Ty1 against SARS-CoV-2 pseudovirus}

The stoichiometry and kinetics of a target-binding interaction can be favorably influenced by three general approaches: (i) increasing the molar abundance and the soluble antigen concentration, (ii) improving the binding interaction affinity under relevant assay conditions, and (iii) rising the capturing reagents (e.g. antibodies) abundance and concentration through surface immobilization according to the law of mass action (Esteban Fernández de Ávila et al., 2013; Miller et al., 2018). Since it is not practical to raise the concentration of antigens or the affinity of already optimized antibodies, here we sought to explore the third strategy of increasing the surface densities of Ty1-CBD via immobilization on cellulose paper. Of note, use of CBD fusion proteins has been demonstrated for the biofunctionalization of cellulose substrates in applications including protein purification (Sugimoto et al., 2012; Tomme et al., 1998), textile manufacturing (Levy \& Shoseyov, 2002), and immunoassay development (Dai et al., 2017; Holstein et al., 2016; Hussack et al., 2009; H.-D. Kim et al., 2013). Notably, the CBD domain can facilitate the absorption of CBD-containing fusion proteins to cellulose in molar quantities, which allows for an excess amount of immobilized proteins relative to the soluble target (Dai et al., 2017; M. Li et al., 2016).

To explore the consequences of enhanced binder immobilization upon target capture efficiency, we evaluated the capability of Ty1-CBD fusion proteins in capturing SARS-CoV-2 mimics (referred to as pseudovirus in this work). One of the gold standards for SARS-CoV-2-related studies is to use non-replicative lentivirus pseudotyped with the S protein derived from SARS-CoV-2 in conjunction with mammalian cells engineered to express human ACE2 (hACE2) (Nie et al., 2020) (Figure 3a ). Since emerging SARS-CoV-2 variants with higher transmission rates bear mutations in the $\mathrm{S}$ protein, one advantage of the pseudovirus system is that it can rapidly evaluate intervention approaches against different spike variants. Using this system, we compared the original wildtype (WT) S spike to the "D614G" mutant, in which the $614^{\text {th }}$ aspartate is converted to glycine in the S protein of SASR-CoV-2. Notably, epidemiology and molecular biology studies have demonstrated that the D614G mutant confers higher transmission and worse symptoms in humans (Volz et al., 2021). Therefore, it is of particular interest to assess our fusion protein strategy in the context of both the wildtype and the D614G variant. As shown in Figure 3b and 3c , after HEK293T-hACE2 cells were transduced with wildtype or D614G pseudotyped lentivirus carrying a green fluorescence protein (GFP) reporter, $\sim 50 \%$ of cells were GFP positive with D614G pseudovirus exhibiting a higher transduction efficacy than that of the wildtype. These findings agreed with the increased infectivity by the D614G mutation (Korber et al., 2020; Zhang et al., 2020). In comparison, transduction of the parental HEK293T cell line (lacking hACE2 expression) with the same SAR-CoV-2 pseudovirus did not result in GFP expression, which validated an ACE2-dependent infection by SAR-CoV-2 (Yan et al., 2020).

It is worth noting that the levels of SARS-CoV-2 in COVID-19 patients range from $10^{4}$ to $10^{9}$ copies per ml depending on the type of bodily fluids and degree of the symptoms (Pan et al., 2020; Wölfel et al., 2020). Meanwhile, we calculated the titers of wildtype or D614G pseudotyped lentivirus, and estimated that ${ }^{\sim} 10^{5}$ viral particle particles per $\mathrm{ml}$ were present in the culture media. Therefore, to demonstrate the capability of capturing SARS-CoV-2 at the lower end of the viral titer range for SARS-CoV-2-containing fluids, we further diluted the media to contain approximately $\sim 10^{4}$ pseudovirus copies per $\mathrm{ml}$, and quantified the neutralization efficacy of Ty1-CBD-immobilized cellulose paper (Figure 4a ). In addition, filter paper alone or filter paper coated with Ty1 but lacking the CBD module served as negative controls. The neutralization efficiency of Ty1-CBD-immobilized filter paper was calculated by dividing the titer of media treated with Ty1-CBDimmobilized filter paper or other control groups by the initial viral titer (i.e., without any treatment). Indeed, using media containing wildtype or D614G pseudoviruses (Figure 4b ), Ty1-CBD-immobilized filter paper resulted in $\sim$-fold increase of the neutralization efficacy compared to that of filter paper only, and $\sim 1.5$-fold enhancement over filter paper pre-coated with Ty1 alone. Moreover, filter paper pre-coated with Ty1-CBD 
or Ty1 displayed 1.65-fold improvement in neutralizing pseudovirus from the media over free proteins (Ty1CBD or Ty1) at equal concentrations, which indicated that the surface immobilization itself can facilitate target recognition.

\section{Integration of the bifunctional protein with an amorphous cellulose column further enhanced the capture efficiency}

Having validated the increased capture efficiency of fusion proteins immobilized on filter paper, we further sought to enhance the neutralization efficiency by incorporating the fusion proteins into regenerated amorphous cellulose (RAC). Because RAC has been shown to exhibit higher surface area per unit mass than filter paper (Yang et al., 2020), we reasoned that using RAC can increase the immobilization density of Ty1-CBD on cellulose, which in turn improves the rate and the degree of target capture based on the theoretical modeling by others (S. Kim et al., 2021). Moreover, this strategy can potentially expand the utility of Ty1CBD by packing Ty1-CBD-functionalized RAC in a column, which allows for an affinity chromatography system to reduce viral load from contaminated fluids (e.g., blood and saliva) in a continuous mode. To test our hypotheses, we filled a $1 \mathrm{~mL}$ gravity-based column with $0.1 \mathrm{~mL}(\sim 50 \mathrm{mg}$ dry weight) of RAC, which was subsequently saturated with purified Ty1-CBD proteins or E. coli lysate containing the fusion proteins (Figure 4c ). After culture media containing wildtype or D614 pseudoviruses were passed through the functionalized column by gravity, viral titers were determined for different flow through samples. Compared to RAC alone, Ty1-CBD-immobilized columns increased the neutralization efficacy of wildtype and D614G pseudoviruses by $\sim 3.5$ times and $\sim 8$ times, respectively. In contrast, RAC columns carrying an irrelevant $\mathrm{Nb}$ (caffeine specific) fused with CBD or Ty1 alone failed to further enhance the degree of neutralization compared to that of RAC alone (Figure 4d ). Taken together, we demonstrated that the Ty1-CBD fusion protein can be integrated into an RAC column to markedly increase the neutralization efficiency of SARS-CoV-2 pseudovirus in a highly specific and continuous fashion.

\section{Discussion}

In comparison to others' efforts in the development of cost-effective point-of-care (POC) diagnostics, preventative measures, and therapeutic strategies against COVID-19, we have developed a bifunctional fusion protein technology that features SARS-CoV-2 capture from cellulose substrates. Since cellulose represents the most abundant and commonly used biopolymer, our approach holds the promise to enable cellulose-based POC diagnostics, functionalized face masks to reduce airborne virus transmission, and customized affinity columns to decontaminate fluids containing SARS-CoV-2 (Valera et al., 2021). Notably, a similar strategy has been proposed for SARS-CoV-2 detection through cellulose filter paper immobilized with CBD fusion proteins (S. Kim et al., 2021). In their approach, the nucleocapsid protein was fused with the CBD and the detection of SARS-CoV-2 required an antibody to capture viral particles in a "sandwich" format. In comparison, we have developed a single agent by directly linking CBD with a Nb specific for SARS-CoV-2, which may be more convenient and cost effective. Additionally, we have demonstrated that the bifunctional fusion proteins can serve as an affinity agent in the customized cellulose column to deplete virus from fluids. Future work can compare these two approaches in terms of capture efficiency and specificity. In addition, we showed that $\sim 50 \mathrm{mg}$ of Ty1-CBD was produced from one-liter bacterial culture in a shake-flask mode, which in theory could functionalize cellulose paper with a surface area of $0.1 \mathrm{~m}^{2}$ based on our titration experiments. Future work can exploit advanced fermentation technologies to improve the production yield of the fusion proteins.

While this study only focused on Ty1, a recently developed $\mathrm{Nb}$ against the spike protein of SARS-CoV2 (Hanke et al., 2020), the conceptual framework can be easily adapted to target other emerging viruses by substituting the $\mathrm{Nb}$ module with other target-specific Nbs. Moreover, since many other SARS-CoV-2specific Nbs have been identified through immunization and the phage display to target different epitopes for SARS-CoV-2, future work may investigate a combination of CBD fusion proteins comprising different Nbs in a multivalent manner to enhance the capture efficiency (Xiang et al., 2020). Despite the promises demonstrated in this study, one limitation is that only pseudovirus-containing culture media were used to characterize the fusion proteins for proof-of-concept. Therefore, it is necessary to further evaluate our 
approach in real specimens such as blood from COVID-19 patients in the near future.

\section{Acknowledgements}

This work was supported by the Northeastern University COVID19 Rapid Seed Grant (JL), Peer Reviewed Medical Research Program from the Department of Defense's Congressionally Directed Medical Research Programs (JL). We extend our appreciation to the Deputyship for Research \& Innovation, Ministry of Education in Saudi Arabia (JL and AAA) for funding this research work. We would like to express our gratitude to Professor Sara Rouhanifard at Northeastern University's Department of Bioengineering for generously sharing her lab's fluorescent microscope; Professor Ke Zhang at Northeastern University's Department of Chemistry for sharing his lab's equipment.

\section{Conflicts of Interest}

The authors declare that there is no conflict of interest.

\section{References}

Bangaru, S., Ozorowski, G., Turner, H. L., Antanasijevic, A., Huang, D., Wang, X., Torres, J. L., Diedrich, J. K., Tian, J.-H., Portnoff, A. D., Patel, N., Massare, M. J., Yates, J. R., Nemazee, D., Paulson, J. C., Glenn, G., Smith, G., \& Ward, A. B. (2020). Structural analysis of full-length SARS-CoV-2 spike protein from an advanced vaccine candidate.Science, 370 (6520), 1089-1094. https://doi.org/10.1126/science.abe1502

Chang, L., Yan, Y., \& Wang, L. (2020). Coronavirus Disease 2019: Coronaviruses and Blood Safety. Transfusion Medicine Reviews ,34 (2), 75-80. https://doi.org/10.1016/j.tmrv.2020.02.003

Crawford, K. H. D., Eguia, R., Dingens, A. S., Loes, A. N., Malone, K. D., Wolf, C. R., Chu, H. Y., Tortorici, M. A., Veesler, D., Murphy, M., Pettie, D., King, N. P., Balazs, A. B., \& Bloom, J. D. (2020). Protocol and Reagents for Pseudotyping Lentiviral Particles with SARS-CoV-2 Spike Protein for Neutralization Assays. Viruses , 12 (5). https://doi.org/10.3390/v12050513

Dai, G., Hu, J., Zhao, X., \& Wang, P. (2017). A colorimetric paper sensor for lactate assay using a cellulose-Binding recombinant enzyme.Sensors and Actuators, B: Chemical , 238 , 138-144. https://doi.org/10.1016/j.snb.2016.07.008

Esteban Fernández de Ávila, B., Watkins, H. M., Pingarrón, J. M., Plaxco, K. W., Palleschi, G., \& Ricci, F. (2013). Determinants of the Detection Limit and Specificity of Surface-Based Biosensors.Analytical Chemistry , 85 (14), 6593-6597. https://doi.org/10.1021/ac4012123

Haghpanah, F., Lin, G., Levin, S. A., \& Klein, E. (2021). Analysis of the potential impact of durability, timing, and transmission blocking of COVID-19 vaccine on morbidity and mortality. EClinicalMedicine ,35 . https://doi.org/10.1016/j.eclinm.2021.100863

Hanke, L., Vidakovics Perez, L., Sheward, D. J., Das, H., Schulte, T., Moliner-Morro, A., Corcoran, M., Achour, A., Karlsson Hedestam, G. B., Hällberg, B. M., Murrell, B., \& McInerney, G. M. (2020). An alpaca nanobody neutralizes SARS-CoV-2 by blocking receptor interaction. Nature Communications , 11 (1), 4420. https://doi.org/10.1038/s41467-020-18174-5

Harmsen, M. M., \& De Haard, H. J. (2007). Properties, production, and applications of camelid single-domain antibody fragments. Applied Microbiology and Biotechnology, 77 (1), 13-22. https://doi.org/10.1007/s00253$007-1142-2$

Hassanzadeh-Ghassabeh, G., Devoogdt, N., De Pauw, P., Vincke, C., \& Muyldermans, S. (2013). Nanobodies and their potential applications. Nanomedicine (London, England) , 8 (6), 1013-1026. https://doi.org/10.2217/nnm.13.86

Holstein, C. A., Chevalier, A., Bennett, S., Anderson, C. E., Keniston, K., Olsen, C., Li, B., Bales, B., Moore, D. R., Fu, E., Baker, D., \& Yager, P. (2016). Immobilizing affinity proteins to nitrocellulose: A 
toolbox for paper-based assay developers. Analytical and Bioanalytical Chemistry , 408 (5), 1335-1346. https://doi.org/10.1007/s00216-015-9052-0

Huo, J., Le Bas, A., Ruza, R. R., Duyvesteyn, H. M. E., Mikolajek, H., Malinauskas, T., Tan, T. K., Rijal, P., Dumoux, M., Ward, P. N., Ren, J., Zhou, D., Harrison, P. J., Weckener, M., Clare, D. K., Vogirala, V. K., Radecke, J., Moynié, L., Zhao, Y., ... Naismith, J. H. (2020). Neutralizing nanobodies bind SARS-CoV-2 spike RBD and block interaction with ACE2. Nature Structural \& Molecular Biology , 27 (9), 846-854. https://doi.org/10.1038/s41594-020-0469-6

Hussack, G., Luo, Y., Veldhuis, L., Hall, J. C., Tanha, J., \& MacKenzie, R. (2009). Multivalent Anchoring and Oriented Display of Single-Domain Antibodies on Cellulose. Sensors , 9 (7), 5351-5367. https://doi.org/10.3390/s90705351

Kim, H.-D., Choi, S.-L., Kim, H., Sohn, J. H., \& Lee, S.-G. (2013). Enzyme-linked assay of cellulose-binding domain functions from Cellulomonas fimi on multi-well microtiter plate. Biotechnology and Bioprocess Engineering , 18 (3), 575-580. https://doi.org/10.1007/s12257-013-0242-3

Kim, S., Hao, Y., Miller, E. A., Tay, D. M. Y., Yee, E., Kongsuphol, P., Jia, H., McBee, M., Preiser, P. R., \& Sikes, H. D. (2021). Vertical Flow Cellulose-Based Assays for SARS-CoV-2 Antibody Detection in Human Serum. ACS Sensors , 6 (5), 1891-1898. https://doi.org/10.1021/acssensors.1c00235

Kirchdoerfer, R. N., Wang, N., Pallesen, J., Wrapp, D., Turner, H. L., Cottrell, C. A., Corbett, K. S., Graham, B. S., McLellan, J. S., \& Ward, A. B. (2018). Stabilized coronavirus spikes are resistant to conformational changes induced by receptor recognition or proteolysis.Scientific Reports , 8 (1), 15701. https://doi.org/10.1038/s41598-018-34171-7

Koenig, P.-A., Das, H., Liu, H., Kümmerer, B. M., Gohr, F. N., Jenster, L.-M., Schiffelers, L. D. J., Tesfamariam, Y. M., Uchima, M., Wuerth, J. D., Gatterdam, K., Ruetalo, N., Christensen, M. H., Fandrey, C. I., Normann, S., Tödtmann, J. M. P., Pritzl, S., Hanke, L., Boos, J., .. Schmidt, F. I. (2021). Structure-guided multivalent nanobodies block SARS-CoV-2 infection and suppress mutational escape.Science, 371 (6530). https://doi.org/10.1126/science.abe6230

Korber, B., Fischer, W. M., Gnanakaran, S., Yoon, H., Theiler, J., Abfalterer, W., Hengartner, N., Giorgi, E. E., Bhattacharya, T., Foley, B., Hastie, K. M., Parker, M. D., Partridge, D. G., Evans, C. M., Freeman, T. M., de Silva, T. I., McDanal, C., Perez, L. G., Tang, H., .. Montefiori, D. C. (2020). Tracking Changes in SARS-CoV-2 Spike: Evidence that D614G Increases Infectivity of the COVID-19 Virus. Cell , 182 (4), 812-827.e19. https://doi.org/10.1016/j.cell.2020.06.043

Krause, P. R., Fleming, T. R., Longini, I. M., Peto, R., Briand, S., Heymann, D. L., Beral, V., Snape, M. D., Rees, H., Ropero, A.-M., Balicer, R. D., Cramer, J. P., Muñoz-Fontela, C., Gruber, M., Gaspar, R., Singh, J. A., Subbarao, K., Van Kerkhove, M. D., Swaminathan, S., .. Henao-Restrepo, A.-M. (2021). SARS-CoV-2 Variants and Vaccines.New England Journal of Medicine , 385 (2), 179-186. https://doi.org/10.1056/NEJMsr2105280

Lai, S., Ruktanonchai, N. W., Zhou, L., Prosper, O., Luo, W., Floyd, J. R., Wesolowski, A., Santillana, M., Zhang, C., Du, X., Yu, H., \& Tatem, A. J. (2020). Effect of non-pharmaceutical interventions to contain COVID-19 in China. Nature , 585 (7825), 410-413. https://doi.org/10.1038/s41586-020-2293-x

Lan, J., Ge, J., Yu, J., Shan, S., Zhou, H., Fan, S., Zhang, Q., Shi, X., Wang, Q., Zhang, L., \& Wang, X. (2020). Structure of the SARS-CoV-2 spike receptor-binding domain bound to the ACE2 receptor. Nature ,581 (7807), 215-220. https://doi.org/10.1038/s41586-020-2180-5

Levy, I., \& Shoseyov, O. (2002). Cellulose-binding domains: Biotechnological applications. Biotechnology Advances ,20 (3-4), 191-213. https://doi.org/10.1016/s0734-9750(02)00006-x

Li, M., Yue, Y., Zhang, Z.-J., Wang, Z.-Y., Tan, T.-W., \& Fan, L.-H. (2016). Site-Specific and High-Loading 
Immobilization of Proteins by Using Cohesin-Dockerin and CBM-Cellulose Interactions.Bioconjugate Chemistry , 27 (7), 1579-1583. https://doi.org/10.1021/acs.bioconjchem.6b00282

Li, W., Moore, M. J., Vasilieva, N., Sui, J., Wong, S. K., Berne, M. A., Somasundaran, M., Sullivan, J. L., Luzuriaga, K., Greenough, T. C., Choe, H., \& Farzan, M. (2003). Angiotensin-converting enzyme 2 is a functional receptor for the SARS coronavirus. Nature ,426 (6965), 450-454. https://doi.org/10.1038/nature02145

Liu, Y.-C., Kuo, R.-L., \& Shih, S.-R. (2020). COVID-19: The first documented coronavirus pandemic in history. Biomedical Journal ,43 (4), 328-333. https://doi.org/10.1016/j.bj.2020.04.007

Luan, J., Lu, Y., Jin, X., \& Zhang, L. (2020). Spike protein recognition of mammalian ACE2 predicts the host range and an optimized ACE2 for SARS-CoV-2 infection. Biochemical and Biophysical Research Communications , 526 (1), 165-169. https://doi.org/10.1016/j.bbrc.2020.03.047

Miller, E. A., Baniya, S., Osorio, D., Al Maalouf, Y. J., \& Sikes, H. D. (2018). Paper-based diagnostics in the antigen-depletion regime: High-density immobilization of rcSso7d-cellulose-binding domain fusion proteins for efficient target capture. Biosensors and Bioelectronics , 102 , 456-463. https://doi.org/10.1016/j.bios.2017.11.050

Morawska, L., \& Cao, J. (2020). Airborne transmission of SARS-CoV-2: The world should face the reality. Environment International ,139 , 105730. https://doi.org/10.1016/j.envint.2020.105730

Nie, J., Li, Q., Wu, J., Zhao, C., Hao, H., Liu, H., Zhang, L., Nie, L., Qin, H., Wang, M., Lu, Q., Li, X., Sun, Q., Liu, J., Fan, C., Huang, W., Xu, M., \& Wang, Y. (2020). Establishment and validation of a pseudovirus neutralization assay for SARS-CoV-2. Emerging Microbes $\&$ Infections , 9 (1), 680-686. https://doi.org/10.1080/22221751.2020.1743767

Ong, S. W. X., Tan, Y. K., Chia, P. Y., Lee, T. H., Ng, O. T., Wong, M. S. Y., \& Marimuthu, K. (2020). Air, Surface Environmental, and Personal Protective Equipment Contamination by Severe Acute Respiratory Syndrome Coronavirus 2 (SARS-CoV-2) From a Symptomatic Patient. JAMA ,323 (16), 1610-1612. https://doi.org/10.1001/jama.2020.3227

Pan, Y., Zhang, D., Yang, P., Poon, L. L. M., \& Wang, Q. (2020). Viral load of SARS-CoV-2 in clinical samples. The Lancet Infectious Diseases , 20 (4), 411-412. https://doi.org/10.1016/S1473-3099(20)30113-4

Ragan, I., Hartson, L., Pidcoke, H., Bowen, R., \& Goodrich, R. (2020). Pathogen reduction of SARSCoV-2 virus in plasma and whole blood using riboflavin and UV light. PLoS ONE, 15 (5). https://doi.org/10.1371/journal.pone.0233947

Schoeman, D., \& Fielding, B. C. (2019). Coronavirus envelope protein: Current knowledge. Virology Journal , 16 (1), 69. https://doi.org/10.1186/s12985-019-1182-0

Schoof, M., Faust, B., Saunders, R. A., Sangwan, S., Rezelj, V., Hoppe, N., Boone, M., Billesbølle, C. B., Puchades, C., Azumaya, C. M., Kratochvil, H. T., Zimanyi, M., Deshpande, I., Liang, J., Dickinson, S., Nguyen, H. C., Chio, C. M., Merz, G. E., Thompson, M. C., ... Manglik, A. (2020). An ultrapotent synthetic nanobody neutralizes SARS-CoV-2 by stabilizing inactive Spike. Science, 370 (6523), 1473-1479. https://doi.org/10.1126/science.abe3255

Steeland, S., Vandenbroucke, R. E., \& Libert, C. (2016). Nanobodies as therapeutics: Big opportunities for small antibodies. Drug Discovery Today , 21 (7), 1076-1113. https://doi.org/10.1016/j.drudis.2016.04.003

Sternberg, A., \& Naujokat, C. (2020). Structural features of coronavirus SARS-CoV-2 spike protein: Targets for vaccination.Life Sciences , 257, 118056. https://doi.org/10.1016/j.lfs.2020.118056

Sugimoto, N., Igarashi, K., \& Samejima, M. (2012). Cellulose affinity purification of fusion proteins tagged with fungal family 1 cellulose-binding domain. Protein Expression and Purification ,82 (2), 290-296. https://doi.org/10.1016/j.pep.2012.01.007 
Sun, X., Ni, Y., He, Y., Yang, M., Tani, T., Kitajima, S., Barbie, D. A., \& Li, J. (n.d.). Engineering the Immune Adaptor Protein STING as a Functional Carrier. Advanced Therapeutics , n/a (n/a), 2100066. https://doi.org/10.1002/adtp.202100066

Tomme, P., Boraston, A., McLean, B., Kormos, J., Creagh, A. L., Sturch, K., Gilkes, N. R., Haynes, C. A., Warren, R. A., \& Kilburn, D. G. (1998). Characterization and affinity applications of cellulosebinding domains. Journal of Chromatography. B, Biomedical Sciences and Applications , 715 (1), 283-296. https://doi.org/10.1016/s0378-4347(98)00053-x

Valera, E., Jankelow, A., Lim, J., Kindratenko, V., Ganguli, A., White, K., Kumar, J., \& Bashir, R. (2021). COVID-19 Point-of-Care Diagnostics: Present and Future. ACS Nano , 15 (5), 7899-7906. https://doi.org/10.1021/acsnano.1c02981

Volz, E., Hill, V., McCrone, J. T., Price, A., Jorgensen, D., O’Toole, Á., Southgate, J., Johnson, R., Jackson, B., Nascimento, F. F., Rey, S. M., Nicholls, S. M., Colquhoun, R. M., da Silva Filipe, A., Shepherd, J., Pascall, D. J., Shah, R., Jesudason, N., Li, K., .. Connor, T. R. (2021). Evaluating the Effects of SARS-CoV-2 Spike Mutation D614G on Transmissibility and Pathogenicity. Cell , 184 (1), 64-75.e11. https://doi.org/10.1016/j.cell.2020.11.020

Voutilainen, S. P., Nurmi-Rantala, S., Penttilä, M., \& Koivula, A. (2014). Engineering chimeric thermostable GH7 cellobiohydrolases in Saccharomyces cerevisiae. Applied Microbiology and Biotechnology ,98 (7), 29913001. https://doi.org/10.1007/s00253-013-5177-2

Wölfel, R., Corman, V. M., Guggemos, W., Seilmaier, M., Zange, S., Müller, M. A., Niemeyer, D., Jones, T. C., Vollmar, P., Rothe, C., Hoelscher, M., Bleicker, T., Brünink, S., Schneider, J., Ehmann, R., Zwirglmaier, K., Drosten, C., \& Wendtner, C. (2020). Virological assessment of hospitalized patients with COVID-2019. Nature ,581 (7809), 465-469. https://doi.org/10.1038/s41586-020-2196-x

Wrapp, D., Wang, N., Corbett, K. S., Goldsmith, J. A., Hsieh, C.-L., Abiona, O., Graham, B. S., \& McLellan, J. S. (2020). Cryo-EM structure of the 2019-nCoV spike in the prefusion conformation. Science ,367 (6483), 1260-1263. https://doi.org/10.1126/science.abb2507

Xiang, Y., Nambulli, S., Xiao, Z., Liu, H., Sang, Z., Duprex, W. P., Schneidman-Duhovny, D., Zhang, C., \& Shi, Y. (2020). Versatile and multivalent nanobodies efficiently neutralize SARS-CoV-2.Science, 370 (6523), 1479-1484. https://doi.org/10.1126/science.abe4747

Xu, J., Xu, K., Jung, S., Conte, A., Lieberman, J., Muecksch, F., Lorenzi, J. C. C., Park, S., Schmidt, F., Wang, Z., Huang, Y., Luo, Y., Nair, M. S., Wang, P., Schulz, J. E., Tessarollo, L., Bylund, T., Chuang, G.Y., Olia, A. S., ... Casellas, R. (2021). Nanobodies from camelid mice and llamas neutralize SARS-CoV-2 variants.Nature , 595 (7866), 278-282. https://doi.org/10.1038/s41586-021-03676-z

Yan, R., Zhang, Y., Li, Y., Xia, L., Guo, Y., \& Zhou, Q. (2020). Structural basis for the recognition of SARS-CoV-2 by full-length human ACE2. Science , 367 (6485), 1444-1448. https://doi.org/10.1126/science.abb2762

Yang, M., Zhu, G., Korza, G., Sun, X., Setlow, P., \& Li, J. (2020). Engineering Bacillus subtilis as a Versatile and Stable Platform for Production of Nanobodies. Applied and Environmental Microbiology ,86 (8). https://doi.org/10.1128/AEM.02938-19

Zhang, L., Jackson, C. B., Mou, H., Ojha, A., Peng, H., Quinlan, B. D., Rangarajan, E. S., Pan, A., Vanderheiden, A., Suthar, M. S., Li, W., Izard, T., Rader, C., Farzan, M., \& Choe, H. (2020). SARSCoV-2 spike-protein D614G mutation increases virion spike density and infectivity. Nature Communications , 11 (1), 6013. https://doi.org/10.1038/s41467-020-19808-4

Zhao, G., He, L., Sun, S., Qiu, H., Tai, W., Chen, J., Li, J., Chen, Y., Guo, Y., Wang, Y., Shang, J., Ji, K., Fan, R., Du, E., Jiang, S., Li, F., Du, L., \& Zhou, Y. (2018). A Novel Nanobody Targeting Middle East Respiratory Syndrome Coronavirus (MERS-CoV) Receptor-Binding Domain Has Potent Cross- 
Neutralizing Activity and Protective Efficacy against MERS-CoV. Journal of Virology, 92 (18), e00837-18. https://doi.org/10.1128/JVI.00837-18

Zhou, P., Yang, X.-L., Wang, X.-G., Hu, B., Zhang, L., Zhang, W., Si, H.-R., Zhu, Y., Li, B., Huang, C.-L., Chen, H.-D., Chen, J., Luo, Y., Guo, H., Jiang, R.-D., Liu, M.-Q., Chen, Y., Shen, X.-R., Wang, X., ... Shi, Z.-L. (2020). A pneumonia outbreak associated with a new coronavirus of probable bat origin. Nature, 579 (7798), 270-273. https://doi.org/10.1038/s41586-020-2012-7

\section{Figure Legends:}

Figure 1: Development of a bifunctional fusion protein to enable cellulose immobilization and subsequent neutralization of severe acute respiratory syndrome coronavirus 2 (SARSCoV-2). a ) An alpaca-derived high-affinity nanobody (Nb), Ty1 for the receptor-binding domain (RBD) of SARS-CoV-2 was genetically fused with $\mathbf{b}$ ) a thermostable cellulose-binding domain (CBD) isolated from a thermophile, $C$. thermocellum . c ) The fusion protein Ty1-CBD was recombinantly expressed in E. coli . d ) The purification of CBD fusion proteins via nickel-nitrilotriacetic acid (Ni-NTA) or direct usage of $E$. coli cell lysate containing the CBD fusion protein for cellulose immobilization. The RBD of SARS-CoV-2 (in grey) bound with $\mathrm{Nb}$ Ty1 (in green) was adapted from the Protein Data Bank (PDB): 6ZXN. e ) As SARS-CoV-2 is transmitted through surface contact, CBD fusion proteins or CBD-contained E. coli cell lysate were immobilized on the surface of cellulose materials, such as cellulose paper, for viral detection and capturing. f ) Since SARS-CoV-2 can also be transmitted through blood products, we also customized Nbdependent regenerated amorphous cellulose (RAC) materials to capture and deplete the virus from bodily fluids.

Figure 2: The fusion protein maintains its activities in binding cellulose and the RBD domain of SARS-CoV-2 . a ) Detection of immobilized Ty1-CBD on a cellulose paper. Ty1-CBD was first spotted on a piece of cellulose paper. Upon air drying, the paper was incubated with a rat antibody against the FLAG epitope (DYKDDDDK), followed by an anti-rat secondary antibody conjugated with HRP. The dark precipitate "Anti-COVID" was visualized after incubation with 3,3'-Diaminobenzidine (DAB). b ) Quantification of maximal protein absorption on Waterman filter paper. $10 \mu \mathrm{L}$ of serially diluted fusion protein solutions were applied to the filter paper, followed by immunoblotting with anti-FLAG directly on the filter paper. Based on the normalized unit intensity quantified by ImageJ, protein abundance increased with concentration. We estimate that $500 \mathrm{ng}$ of Ty1-CBD binds to $1 \mathrm{~mm}^{2}$ of cellulose paper in saturation status.c ) Schematic of an immunoassay to evaluate the function of the fusion protein. Ty1-CBD fusion proteins were immobilized on cellulose paper and then submerged in culture media containing RBD-Fc $\left({ }^{\sim} 100\right.$ $\mathrm{ng} / \mathrm{ml}$ ) as a proxy for actual SARS-CoV-2. The capturing capability was confirmed by anti-human Fc-HRP and the DAB substrate. The structure of the RBD was adapted from PDB: 6ZXN.d ) Testing the ability of protein-coated cellulose paper discs in capturing RBD-containing media. Representative discs were prepared by a $6-\mathrm{mm}$ biopsy punch and then coated with E. coli lysates containing indicated recombinant fusion proteins. The functionalized discs were incubated with RBD-containing or control (no RBD) media. The intensity of dark staining was strongest from the combination of Ty1-CBD-coated disc and RBD-containing media $(\sim 100 \mathrm{ng} / \mathrm{ml})$.

Figure 3: Generation of wildtype and D614G pseudoviruses for neutralization assays by Ty1CBD-functionalized cellulose . a ) Schematic overview of the pseudotyped virus production: HEK293T cells were transfected with a lentiviral vector expressing a green fluorescent protein (GFP), a plasmid encoding SARS-CoV-2 spike, and packaging vectors. The transfected cells produced lentiviral particles pseudotyped with the S protein of SARS-CoV-2, and the pseudovirus can transduce HEK293T expressing human angiotensin-converting enzyme 2 (hACE2) to express GFP. b ) Microscope images showing that the HEK293T-hACE2 cells expressed GFP after transduction with lentivirus pseudotyped with the wildtype (WT) SARS-CoV-2 spike protein or the D614G variant. Scale bar $=100 \mu \mathrm{m}$. c ) Representative flow cytometric analysis evaluating the transduction efficiency of SARS-CoV-2 WT and D614G pseudoviruses compared with two negative control groups: HEK293T-hACE2 without any transduction and HEK293T transduced with SARS-CoV-2 WT pseudotyped lentivirus. Results are representative of three independent 
experiments.

Figure 4: SARS-CoV-2 pseudovirus capture by Ty-CBD-immobilized cellulose in two different formats: a ) Schematic of increasing surface densities of Ty1-CBD through protein immobilization on cellulose materials for SARS-CoV-2 neutralization. b ) Increased neutralization efficacy of pseudovirus through protein immobilization on cellulose paper over free proteins. After incubating the pseudovirus with $200 \mu \mathrm{L}$ of $10 \mu \mathrm{g} / \mathrm{ml}$ fusion protein Ty1-CBD or Ty1 (negative control) immobilized on cellulose paper or free protein with equal concentrations, the titers of wildtype (WT) and D614G pseudoviruses were quantified by transducing HEK293T-hACE2 cells with the remaining viruses in the supernatant. Fold changes from each treatment group were normalized to that of filter paper only. c ) A Ty1-CBD-functionalized RAC column to capture the antigen of interest in a continuous fashion. d ) Neutralization efficacy of Ty1-CBD-functionalized RAC. The flow through samples from functionalized RAC columns were used to transduce HEK293T-hACE2 cells to quantify viral titers for WT and D614G SARS-CoV-2 pseudoviruses, respectively. Fold changes from each treatment group were normalized to that of RAC only. Graphs are expressed as mean \pm SEM $(n=4)$ in $\mathbf{b}$ and as mean \pm SEM $(n=3)$ in $\mathbf{d}$. Statistical analysis was performed by one-way analysis of variance (ANOVA) according to the following scale: ${ }^{* *} P<0.01,{ }^{* * *} P<0.001$, and ${ }^{* * * *} P<0.0001$.

Figure 1

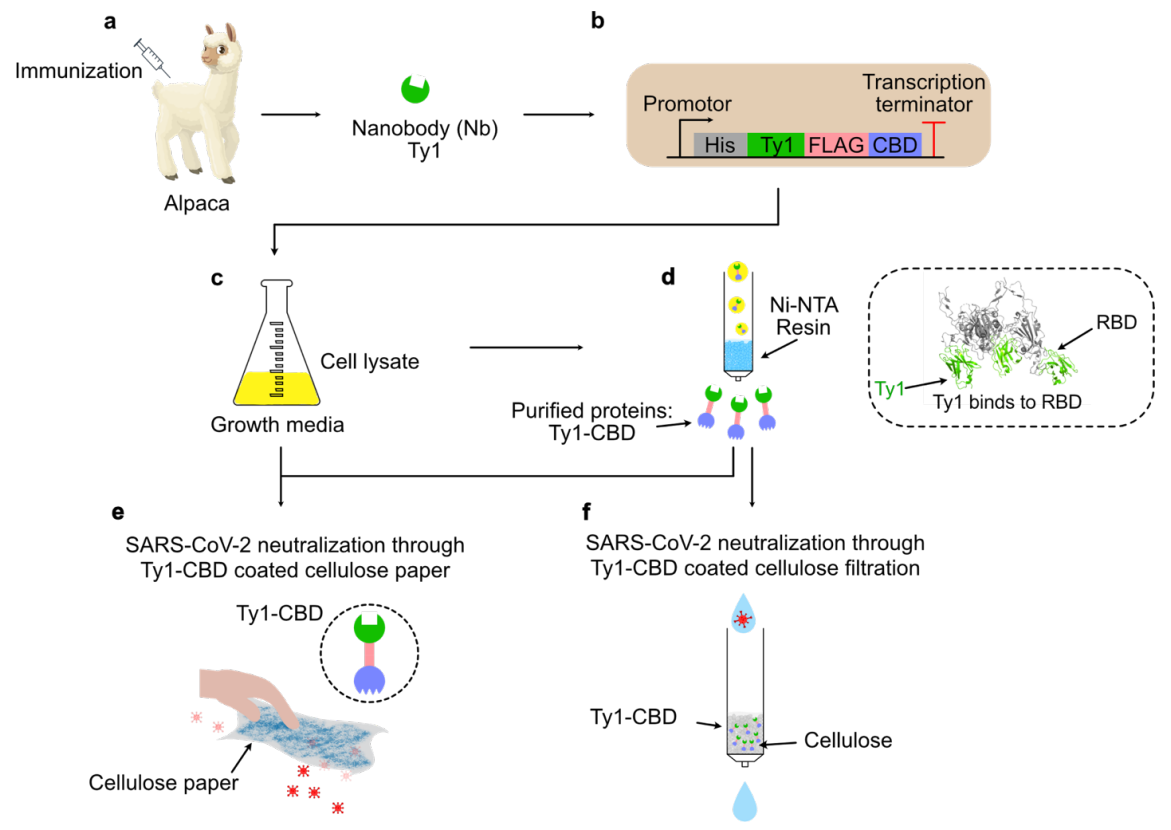

Figure 2 
a

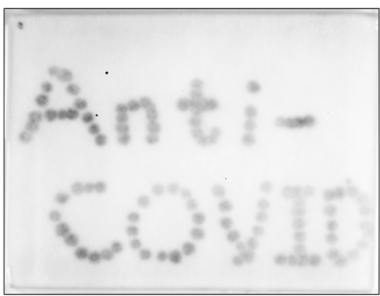

c
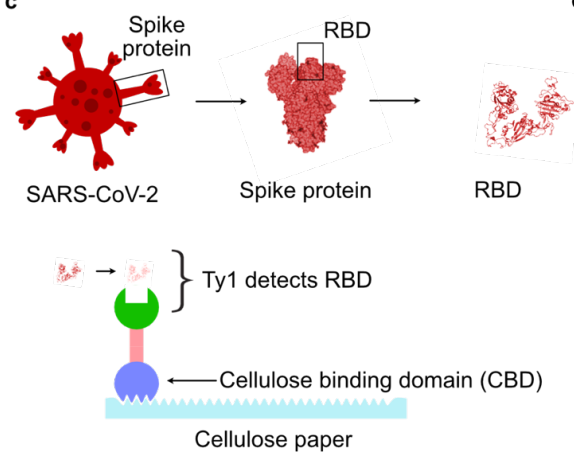

b

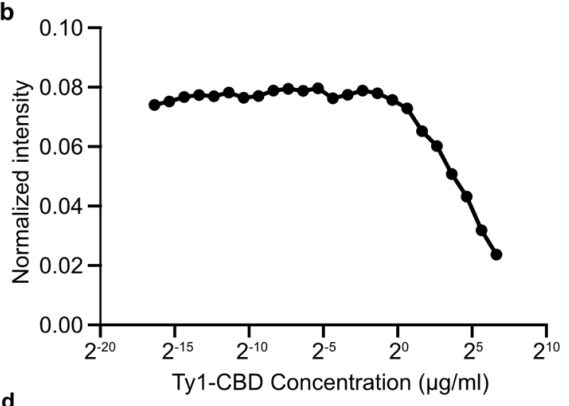

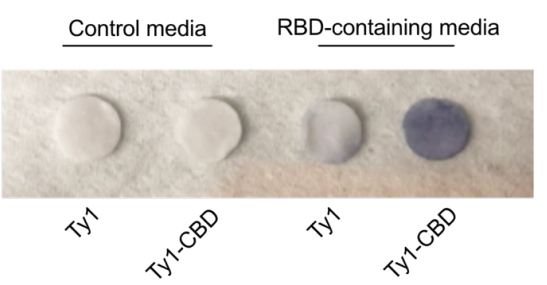

Figure 3

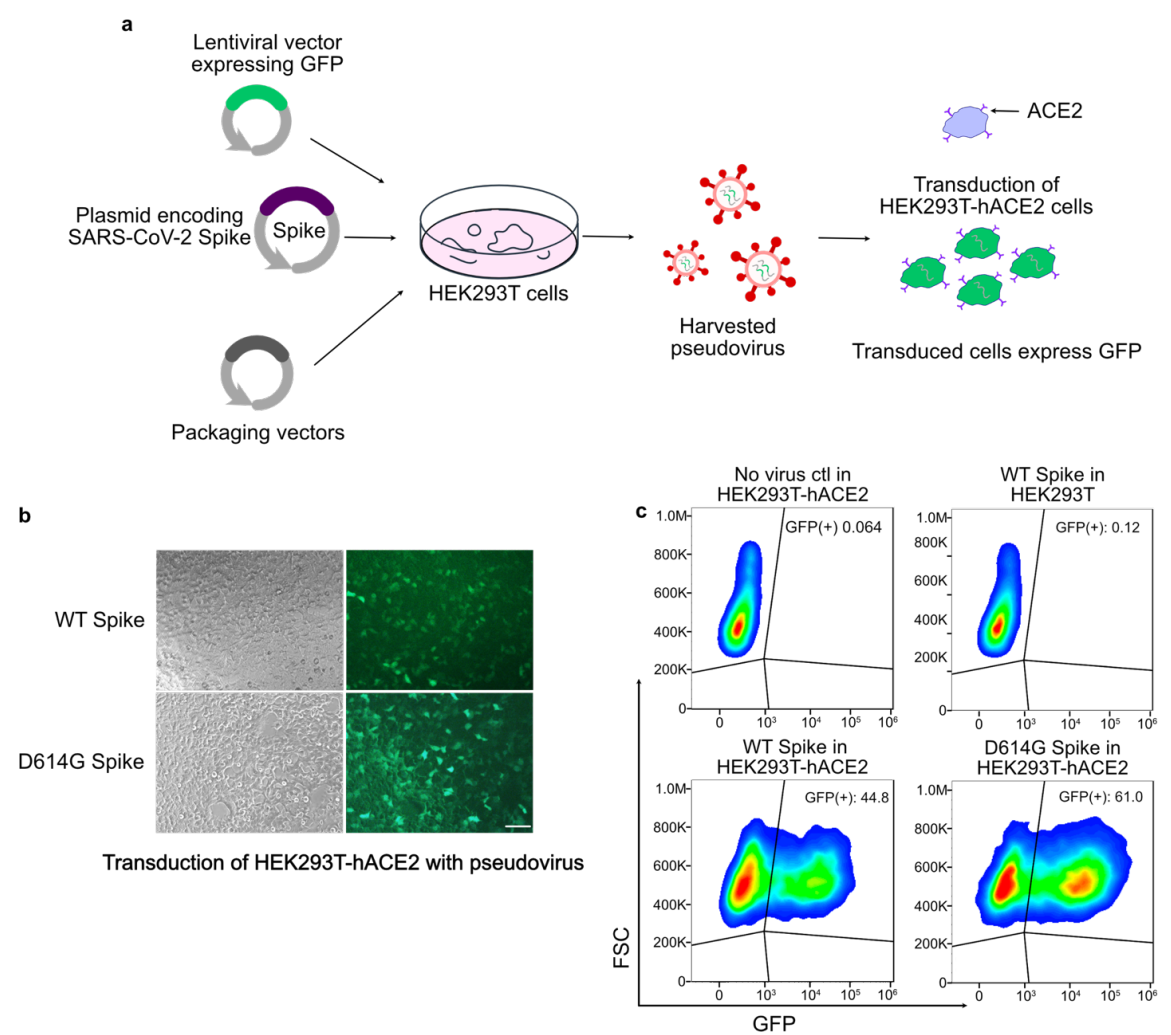

Figure 4 

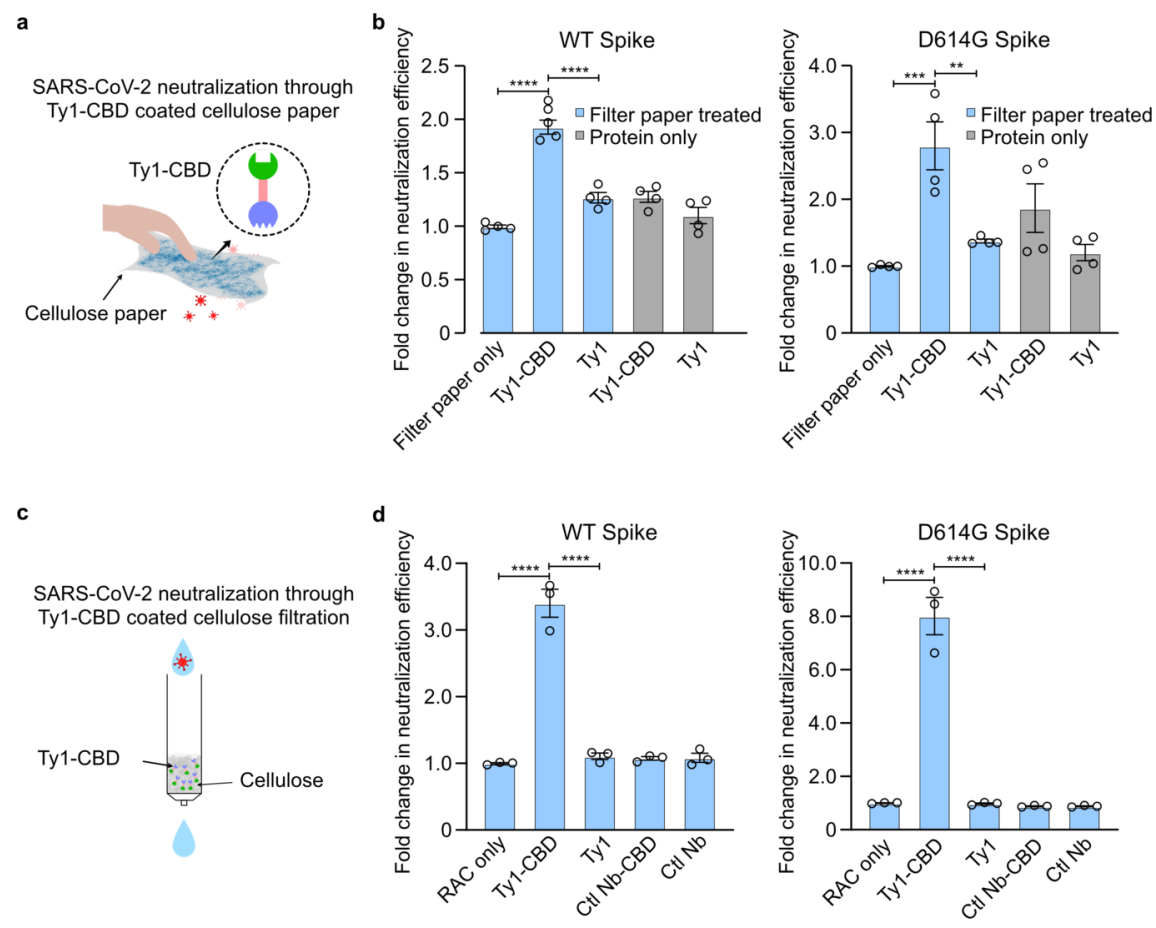

Supplementary Materials

Nanobody-Functionalized Cellulose for Capturing and Containing SARS-CoV-2

Xin Sun ${ }^{1}$, Shaobo Yang ${ }^{1}$, Amal A. Al-Dossary ${ }^{2}$, Shana Broitman ${ }^{1}$, Yun Ni ${ }^{1}$, Mengdi Yang ${ }^{1}, \mathrm{Jiahe}^{1}{ }^{*}$

1Department of Bioengineering, Northeastern University, Boston, MA, United States, $02115^{2}$ Department of Basic Sciences, Deanship of Preparatory Year and Supporting Studies, Imam Abdulrahman Bin Faisal University, Dammam, Saudi Arabia, 34212

Corresponding author: Jiahe Li, email: jiah.li@northeastern.edu

Supplemental Figures 


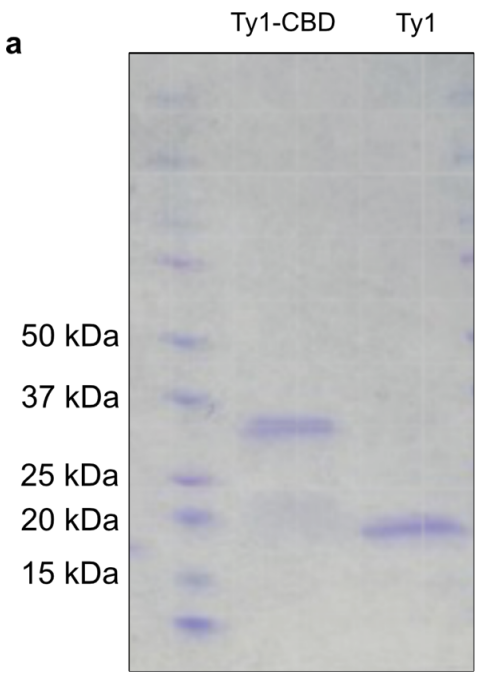

b

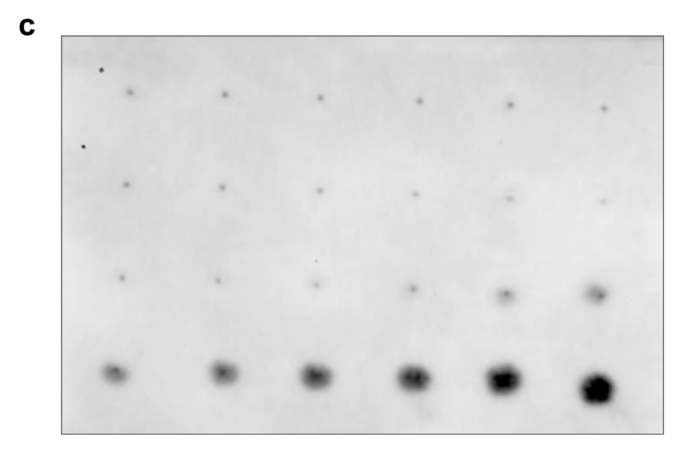

Figure S1 a a ) Denaturing SDS-PAGE of Ty1 and Ty1-CBD. b ) Size exclusion chromatography of Ty1 and Ty1-CBD.c ) Quantification of maximum binding capacity per unit area through spotting serially diluted Ty1-CBD proteins on cellulose paper. Ty1-CBD proteins were detected by direct immunoblotting on cellulose paper via an anti-FLAG antibody.
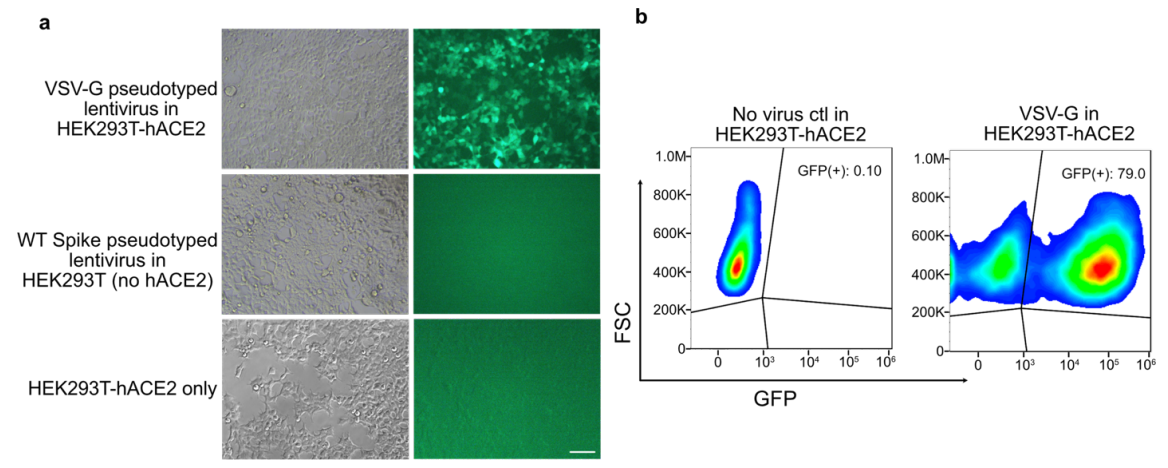

Figure S2 . a ) Microscopy images of HEK293T-hACE2 cells transduced with lentivirus pseudotyped with vesicular stomatitis virus G (VSV G) protein (first panel); HEK293T transduced with lentivirus pseudotyped with the SARS-CoV-2 WT Spike (second panel); and HEK293T-hACE2 without any transduction (third panel). Scale bar $=100 \mu \mathrm{m}$. b ) Flow cytometry of GFP expression from VSV-G pseudotyped lentivirus in HEK293T-hACE2 cells. Results are representative of three independent experiments. 